\title{
MORBILIDAD Y MORTALIDAD EN EL INSTITUTO MATERNO-INFANTIL "CONCEPCION VILLAVECES DE ACOSTA" Durante los años 1965 y 1966
}

\author{
Dr. Rafael López Ruiz*
}

Para este estudio, así como para los de los años anteriores (hemos venido haciéndolo desde 1960) nos hemos basado, antes que todo, en las Historias Clínicas. Este documento hospitalario es el fundamento de toda información asistencial, científica, y administrativa. Por ello recordamos su importancia y volvemos a afirmar que el máximo cuidado de directores, profesores y administradores hospitalarios debe ser el de la Historia Clínica.

En los Establecimientos asistenciales de San Juan de Dios usamos la Nomenclatura "Standard" de la Asociación Médica Americana y el Método de Clasificación Estadística Internacional de la Organización Mundial de la Salud, para la recopilación de los diagnósticos. La nomenclatura Standard de la A.M.A. comprende, como todos saben, dos grupos de dígitos separados por un guión. En la primera parte está encerrada toda topografía y en la segunda toda etiología conocida o posible. Por ejemplo: Fracturas de Icuello del fémur tendrá el índice siguiente: 23514-416. Y de esa manera todas las localizaciones anatómicas conocidas y todas las etiologías tienen un número invariable y por consiguiente único y dife- rente para cada caso. Así, cuando se emprende una investigación se solicita en la Oficina de Estadística el índice correspondiente al tema y allí se encuentran los números de las historias atañederas y nada más que los de esas historias. El investigador está seguro de encontrar entre los documentos que se le suministran los pertinentes y seguro también, por tanto, de no perder tiempo escogiendo o separando documentos que no le conciernen.

La clasificación estadística de la $\mathrm{O}$. M.S. es de grupo, es decir, que aglutina en un solo haz varias topogragías similares.

Acontece, entonces, que cuando se busca respuesta a interrogantes comprendidos dentro de uno de los grupos, siempre van muchas historias que no tienen relación precisa con el tema solicitado y el investigador debe, claro está, gastar tiempo seleccionando y apartando las de su preferencia. Sin embargo esta clasificación Estadística Internacional O.M.S. cuya metodoolgía fue basada en las conclusiones definitivas de varios congresos internacionales inspirados $y$

\footnotetext{
* Jefe de Estadística Científica de los Establecimientos Hospitalarios de San Juan de Dios.
} 
orientados ellos en la sabiduría ofrecida por la NOSOLOGIA METHODICA de Francois Bossier de la Croix, por la obra del gran Lineo GENERA MORBORUM y la notable clasificación de Guillen SYNOPSIS NOSOLOGIAE METHODICAE, es irreemplazable para los informes y los estudios de conjunto, precisamente porque reduce a grupos bien determinados todo el acervo investigativo. Así pues; la tabulación, la numeración, totalización y comparación son mucho más cómodas. La O.M.S. ha dividido la morbilidad y las causas de defunción en XVII grupos y estos son los que hemos seguido para la presentación de nuestro estudio de conjunto.

\section{GRUPOS DE LA O.M.S.}

I Enfermedades infecciosas y parasitarias.

II Neoplasmas malignos y benignos.

III Alérgicas, endocrinas y nutricionales.

IV Sangre y hematopoyéticos.

$\mathrm{V}$ Mentales y Psiconeurosis.

VI Nerviosas y órganos de los sentidos.

VII Enfermedades circulatorias.

VIII Enfermedades respiratorias.

IX Enfermedades digestivas.

$X \quad$ Enfermedades génito urinarias.

XI Embarazo, parto, puerperio.

XII Enfermedades cutáneas.

XIII Huesos, músculos, locomoción.

XIV Enfermedades congénitas.

XV Enfermedades de la primera infancia.

$X V I$ Síntomas $y$ síndromes.

XVII Traumatismos, violencias, envenenamientos.

Y Clasificación suplementaria especial.

Estos grupos $\mathbf{Y}$ están destinados a la tabulación de vacunaciones y otros estados íntimamente relacionados con los estudios de morbilidad y mortalidad o de condiciones sanitarias determinadas.
La Tabla - A - Sinopsis del movimiento científico asistencial, destaca cifras de alta significación tales como 19.074 partos para 1965 y 20.326 para 1966, los abortos fueron 4.738 en 1965 y 5.555 en 1966, el total de entradas en 1965 dio 26.568 y en 1966 28.510. Muchos otros renglones de esta Tabla acusa nun aumento creciente del año anterior al siguiente $y$ si consideramos que el Instituto $\mathrm{Ma}$ terno Infantil solo cuenta con 365 camas adultas y 191 infantiles y, además, que es la única Institución que sirve gratis a una población de 2'000. 000 de habitantes como la de Bogotá actual, podemos ver con toda exactitud la realidad de la enorme labor asistencial y científica que cumple a cabalidad este benemérito componente de los Establecimientos Hospitalario sde San Juan de Dios.

\section{Morbilidad materna}

Como fuera de los casos obstétricos propiamente dichos se atienden, con relativa frecuencia afecciones varias, las hemos agrupado en los cuadros de morbilidad - TABLA B clasificada según los XVII capítulos de la O.M.S. En estos renglones la renovada lúes y el carcinoma del cérvix hacen un importante acto de presencia lo mismo que las intoxicaciones o envenenamientos de origen exógeno.

Dejamos que el lector saque, de sus reflexiones sobre los cuadros y las cifras explicativas, todas las conclusiones importantes que a su clarividencia no escaparan.

La morbilidad - TABLA C - y la mortalidad - TABLAS D y $E$ - han sido repartidas en los ya tan mencionados XVII grupos de la O.M.S. para hacer verdaderamente objetiva su descripción. De otra manera resultaría inocuo y acusaría pereza investi- 
gativa decir: "la morbilidad y la mortalidad infantiles, suman para los dos años 8.416 casos, más 962 respectivamente". No, preferimos describir esa morbilidad y esa mortalidad en los XVII capítulos ya dichos, en lugar, repetimos, de contentarnos con decir: estos casos están todos comprendidos en el renglón XV consagrado a enfermedades de la primera infancia.

En la - TABLA B - Morbilidad Materna Ilamamos la atención sobre las enfermedades infecciosas, los neoplasmas uterinos, y las circulatorias. Comparando las cifras totales vemos que para 1966, frente a 1965, hubo aumento $y$ ese aumento se expresa así: Infecciosas 26,54\%. Carcinoma del cérvix $3,78 \%$ y Circulatorias $27,19 \%$.

Para los recién nacidos y según se desprenden de las Tablas D y E son fatales las enfermedades respiratorias y las digestivas ya que las cifras pertinentes expuestas acusan a esas dolencias como causantes del mayor número de defunciones.

Complemento indispensable de esta esquemática representación estadística nos parecen las Tablas $F$ y $F$ bis que discriminan las muertes maternas según la historia clínica, la edad y la causa del fallecimiento. Es también utilísimo, creemos nosotros, presentar en las Tablas $G$ y $G$ bis los índices biográficos correspondientes a 1965 y 1966 respectivamente.

Las Tablas $\mathrm{H}$ y $\mathrm{H}$ bis podrán satisfacer la curiosidad en lo relacionado con porcentajes de permanencia, de ocupación de camas, de toxemias y de mortalidad etc.
Las Tablas I del Dr. Amarís Mora, Jefe del Departamento de Pediatría, muestran el funcionamiento de esos Servicios. Aquí también se registra un aumento apreciable de la atención médica prestada $y$, desde luego, un aumento del trabajo y de la tarea ingente que el médico efectúa en el servicio y cuidado de los pacientes que acuden al Instituto Materno Infantil "Concepción Villaveces de Acosta". En efecto, si consideramos, por ejemplo, que el total de consultas en 1965 fue de 30.142 y en 1966 de 33.179 el porcentaje de aumento señala un $10,7 \%$ si enfrentamos el total de Consultas Externas de 1965 (6.933) con el de 1966 (8.247) hallamos un porcentaje de aumento del tenor de $18,95 \%$. Séanos permitido, entonces, volver a decir que señalamos y destacamos la realidad de la enorme labor asistencial y científica que cumple a cabalidad este benemérito componente de los establecimientos hospitalarios de San Juan de Dios sostenido y llevado de la mano por la Beneficencia de Cundinamarca.

Antes de terminar creemos importante llamar la atención de los investigadores y de los eruditos sobre la extensión del horizonte de búsqueda en cada uno de los grupos O.M.S. particularizado, si es el caso, por la selección de los Indices "Standard" de la Asociación Médica Americana, ya que, repetimos, podemos suministrar todas y cada una de las historias correspondientes al caso en estudio. Hacemos notar también que el número de diagnósticos no significa, exactamente, el mismo número de pacientes, puesto que la unidad diagnóstico no es igual a la unidad paciente ya que un enfermo puede padecer diferentes afecciones $y$ todas, sin excepción, contribuyen a sumar el total de la morbilidad. 
TABLA A

\section{INSTITUTO MATERNO INFANTIL - SINOPSIS DEL MOVIMIENTO CIENTIFICO ASISTENCIAL 1965 y 1966 COMPARATIVAMENTE}

\begin{tabular}{|c|c|c|c|c|}
\hline & 1965 & 1966 & & $+\%$ \\
\hline Entradas & 25.568 & 28.510 & + & $11,52 \%$ \\
\hline Estancias & 117.404 & 94.587 & - & $19,43 \%$ \\
\hline Egresos & 26.754 & 28.391 & + & $6,11 \%$ \\
\hline Partos & 19.074 & 20.326 & + & $6,56 \%$ \\
\hline Nacidos vivos & 18.684 & 19.874 & + & $6,36 \%$ \\
\hline Nacidos muertos & 603 & 644 & + & $6,79 \%$ \\
\hline Cesáreas & 853 & 1.047 & + & $22,74 \%$ \\
\hline Fórceps & 1.151 & 1.342 & + & $16,59 \%$ \\
\hline Abortos atendidos & 4.738 & 5.555 & + & $17,24 \%$ \\
\hline Defunciones maternas & 46 & 76 & + & $65,21 \%$ \\
\hline Defunciones infantiles & 612 & 638 & + & $4,24 \%$ \\
\hline Intervenciones varias & 273 & 306 & + & $12,98 \%$ \\
\hline Consultas prenatales: & & & & \\
\hline de $1 \mathrm{a}$. vez & 5.563 & 6.689 & + & $2,24 \%$ \\
\hline de $2 a$. vez, etc & 13.348 & 16.764 & + & $25,58 \%$ \\
\hline Totales & 18.911 & 23.453 & + & $24,07 \%$ \\
\hline Análisis de Laboratorio & 145.117 & 159.207 & + & $9,70 \%$ \\
\hline Transfusiones & 3.717 & 3.848 & + & $3,52 \%$ \\
\hline Radiografías & 1.561 & 2.111 & + & $35,23 \%$ \\
\hline Radioscopias & 41 & 0 & & 0 \\
\hline \multicolumn{5}{|l|}{ Fórmulas despachadas: } \\
\hline Internas & 4.761 & 4.013 & - & $15,71 \%$ \\
\hline Externas & 57.720 & 41.358 & - & $28,34 \%$ \\
\hline Totales & 62.481 & 45.371 & - & $27,38 \%$ \\
\hline Consultas externas de Puericultura y Pediatría: & & & & \\
\hline de la. vez & 7.542 & 8.252 & + & $9,41 \%$ \\
\hline de $2 a$. vez & 22.600 & 24.927 & + & $10,29 \%$ \\
\hline Totales & 30.142 & 33.197 & + & $10,13 \%$ \\
\hline $\begin{array}{l}\text { Intervenciones quirúrgicas } \\
\text { Infantiles: }\end{array}$ & 125 & 106 & - & $15,20 \%$ \\
\hline
\end{tabular}


TABLA B

INSTITUTO MATERNO INFANTIL "CONCEPCION VILLAVECES DE ACOSTA" MORBILIDAD MATERNA (NO OBSTETRICA) EN EL AÑO 1966. CLASIFICADAS SEGUN LOS GRUPOS PATOLOGICOS ESTADISTICOS DE LA O.M.S. COMPARACION CON EL AÑO 1965

\begin{tabular}{|c|c|c|c|c|c|}
\hline Grupos & Descripción & 1965 & $\%$ & 1966 & $\%$ \\
\hline I. & Infecciones y parasitarias (Lúes $1965=34-1966=45$ ) & 113 & 6,30 & 163 & 8,10 \\
\hline 11. & Neoplasmas (Carcinoma del cérvix, $1965=20-1966=14$ ) & 132 & 7,36 & 137 & 7,81 \\
\hline III. & Alérgicas, endocrinas, nutricionales & 54 & 3,01 & 49 & 2,79 \\
\hline IV. & Sangre y hematopoyéticos & 69 & 3,85 & 15 & 0,85 \\
\hline V. & Mentales y Psiconeurosis & 15 & 0,84 & 11 & 0,62 \\
\hline VI. & Nerviosas y órganos de los sentidos & 34 & 1,90 & 30 & 1,71 \\
\hline VII. & Enfermedades circulatorias & 114 & 6,36 & 145 & 8,27 \\
\hline VIII. & Enfermedades respiratorias & 82 & 4,57 & 94 & 5,36 \\
\hline IX. & Enfermedades digestivas & 178 & 9,93 & 141 & 8,04 \\
\hline$X$ & Génito-urinarias & 501 & 27,94 & 409 & 23,34 \\
\hline$\times 1$ & Embarazo, parto, puerperio (ver los cuadros sinópticos) & & & & \\
\hline XII. & Enfermedades cutáneas & 22 & 1,22 & 11 & 0,51 \\
\hline XIII. & Huesos, músculos, locomoción & 11 & 0,61 & 2 & 0,11 \\
\hline XIV. & Enfermedades congénitas & 12 & 0,67 & 5 & 0,27 \\
\hline XV. & $\begin{array}{l}\text { Enfermedades de la primera infancia (están } \\
\text { distribuídas en los demás grupos }\end{array}$ & & & & \\
\hline XVI. & Síntomas y síndromes & 424 & 23,65 & 518 & 29,56 \\
\hline$X V \|$. & Traumatismos, violencias, envenenamientos & 32 & 1,79 & 42 & 23,97 \\
\hline & TOTALES & 1.793 & & 1.752 & \\
\hline
\end{tabular}

TABLA C

INSTITUTO MATERNO INFANTIL "CONCEPCION VILLAVECES DE ACOSTA"
MORBILIDAD INFANTIL EN EL AÑO 1966. CLASIFICADA SEGUN LOS GRUPOS PATOLOGICOS ESTADISTICOS DE LA O.M.S. COMPARACION CON EL AÑO 1965

\begin{tabular}{|c|c|c|c|c|c|}
\hline Grupos & Descripción & 1965 & $\%$ & 1966 & $\%$ \\
\hline I. & Infecciosas y parasitarias (Lúes 57) & 574 & 14,49 & 264 & 5,92 \\
\hline II. & Neoplasmas & 11 & 0,28 & 5 & $0,1 ?$ \\
\hline$\cdot 111$ & Alérgicas, endocrinas, nutricionales & 123 & 3,11 & 163 & 3,65 \\
\hline IV. & Sangre y hematopoyéticos & 769 & 19,41 & 871 & 19,55 \\
\hline V. & Mentales y Psiconeurosis & 0 & 0,00 & 0 & 0,00 \\
\hline VI. & Nerviosas y órganos de los sentidos & 269 & 6,79 & 596 & 13,37 \\
\hline VII. & Enfermedades circulatorias & 5 & 0,13 & 11 & 0,24 \\
\hline VIII. & Enfermedades respiratorias & 1.360 & 34,43 & 1.182 & 26,53 \\
\hline IX. & Enfermedades digestivas & 258 & 6,51 & 264 & 5,92 \\
\hline$x$ & Génito-urinarias & 6 & 0,15 & 22 & 0,49 \\
\hline XI. & Embarazo, parto, puerperio (ver los cuadros sinópticos) & 0 & 0,00 & 0 & 0,00 \\
\hline XII. & Enfermedades cutáneas & 184 & 4,65 & 449 & 10,07 \\
\hline XIII. & Huesos, músculos, locomoción & 2 & 0,05 & 1 & 0,02 \\
\hline XIV. & Enfermedades congénitas & 291 & 7,35 & 525 & 11,78 \\
\hline XV. & $\begin{array}{l}\text { Enfermedades de la primera infancia (están } \\
\text { distribuídas en los demás grupos }\end{array}$ & & & & \\
\hline XVI. & Síntomas y síndromes & 4 & 0,10 & 12 & 0,26 \\
\hline \multirow[t]{2}{*}{$X V I I}$. & Traumatismos, violencias, envenenamientos & 105 & 2,65 & 90 & 20,20 \\
\hline & $\begin{array}{l}\text { TOTALES } \\
\text { VACUNA B. C. G. }=11.7791965=4.367) \\
\text { NIÑO SANO }=16.488(1965=15.665)\end{array}$ & 3.961 & & 4.455 & \\
\hline
\end{tabular}


TABLA D

INSTITUTO MATERNO INFANTIL "CONCEPCION VILLAVECES DE ACOSTA"

MORTALIDAD NEO-NATAL EN 1966. CLASIFICADA SEGUN LOS GRUPOS ESTADISTICOS

DE LA O.M.S. COMPARACION CON EL AÑO 1965

\begin{tabular}{|c|c|c|c|c|c|c|}
\hline Grupos & Descripción & & 1965 & $\%$ & 1966 & $\%$ \\
\hline 1 & Infecciosas y parasitarias & & 13 & 38,12 & 12 & 33,24 \\
\hline II. & Neoplasmas & & 0 & 0 & 0 & 0 \\
\hline III. & Alérgicas, endocrinas, nutricionales & & 3 & 0,87 & 8 & 22,15 \\
\hline IV. & Sangre y hematopoyéticos & & 74 & 21,70 & 49 & 13,57 \\
\hline V. & Mentales y Psiconeurosis & & 0 & 0 & 0 & 0 \\
\hline VI. & Nerviosas y órganos de los sentidos & & 0 & 0 & 2 & 0,55 \\
\hline VII. & Enfermedades circulatorias & & 0 & 0 & 4 & 11,08 \\
\hline VIII. & Enfermedades respiratorias & & 216 & 63,63 & 225 & 62,32 \\
\hline$\cdot \mathrm{IX}$ & Enfermedades digestivas & & 13 & 3,81 & 4 & 11,08 \\
\hline $\mathrm{X}$. & Génito-urinarias & & 0 & 0 & 0 & 0 \\
\hline $\mathrm{XI}$ & Embarazo, parto, puerperio (ver los cuadros & sinópticos ) & - & - & - & - \\
\hline XII. & Enfermedades cutáneas & & 0 & 0 & 0 & 0 \\
\hline XIII. & Huesos, músculos, locomoción & & 0 & 0 & 0 & 0 \\
\hline XIV. & Enfermedades congénitas & & 12 & 3,51 & 35 & 9,69 \\
\hline$X V$ & $\begin{array}{l}\text { Enfermedades de la primera infancia (están } \\
\text { distribuídas en los demás grupos }\end{array}$ & & - & & & 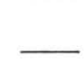 \\
\hline XVII. & Síntomas y síndromes & & 9 & 2,63 & 1 & 0,34 \\
\hline XVI. & Traumatismos, violencias, envenenamientos & & 1 & 0,21 & 2 & 0,55 \\
\hline YOO & Sin dato & & 0 & 0 & 19 & 5,26 \\
\hline \multirow{4}{*}{$1965:$} & \multicolumn{2}{|c|}{ TOTALES } & 341 & \multicolumn{3}{|c|}{361} \\
\hline & \multicolumn{2}{|c|}{$\begin{array}{l}\text { Porcentajes de mortalidad Neo-natal } \\
\text { Defunciones } \\
\qquad 341\end{array}$} & \multicolumn{4}{|c|}{361} \\
\hline & $-------=1,82 \%$ & --- & - & - & $-=$ & $1,81 \%$ \\
\hline & Nacidos vivos & Nacidos vivos & & 19.8 & & \\
\hline
\end{tabular}




\section{TABLA E}

INSTITUTO MATERNO INFANTIL "CONCEPCION VILLAVECES DE ACOSTA" MORTALIDAD NEO-NATAL EN 1966. CLASIFICADA SEGUN LOS GRUPOS ESTADISTICOS DE LA O.M.S. COMPARACION CON EL AÑO 1965

\begin{tabular}{|c|c|c|c|c|c|c|}
\hline Grupos & Descripción & & 1965 & $\%$ & 1966 & $\%$ \\
\hline I. & Infecciosas y parasitarias & & 22 & 18,03 & 54 & 37,21 \\
\hline II. & Neoplasmas & & 0 & 0 & 0 & 0 \\
\hline$\cdot 111$ & Alérgicas, endocrinas, nutricionales & & 5 & 4,09 & 3 & 2,16 \\
\hline IV. & Sangre y hematopoyéticos & & 17 & 13,81 & 6 & 4,34 \\
\hline v. & Mentales y Psiconeurosis & & 0 & 0 & 0 & 0 \\
\hline VI. & Nerviosas y órganos de los sentidos & & 1 & 0,81 & 3 & 2,16 \\
\hline VII. & Enfermedades circulatorias & & 1 & 0,81 & 2 & 1,44 \\
\hline VIII. & Enfermedades respiratorias & & 33 & 27,04 & 42 & 30,44 \\
\hline $\mathrm{IX}$ & Enfermedades digestivas & & 35 & 28,68 & 17 & 12,31 \\
\hline$x$ & Génito-urinarias & & 2 & 1,63 & 1 & 0,72 \\
\hline $\mathrm{XI}$. & Embarazo, parto, puerperio (ver los cuadros & sinópticos ) & 0 & 0 & 0 & 0 \\
\hline XII. & Enfermedades cutáneas & & 0 & 0 & 0 & 0 \\
\hline XIII. & Huesos, músculos, locomoción & & 0 & 0 & 0 & 0 \\
\hline XIV. & Enfermedades congénitas & & 5 & 4,09 & 10 & 7,24 \\
\hline $\mathrm{XV}$ & $\begin{array}{l}\text { Enfermedades de la primera infancia (están } \\
\text { distribuídas en los demás grupos }\end{array}$ & & - & - & - & \\
\hline XVI. & Síntomas y síndromes & & 1 & 0,81 & 0 & 0 \\
\hline XVII. & Traumatismos, violencias, envenenamientos & & 0 & 0 & 0 & 0 \\
\hline \multirow[t]{3}{*}{ YOO } & Sin dato & & - & & - & \\
\hline & TOTALES & & 122 & & 138 & \\
\hline & Porcentajes de mortalidad No-neo-natal & & & & & \\
\hline \multirow[t]{3}{*}{$1965:$} & Defunciones & Defunciones & & 13 & & \\
\hline & $--------=0,65 \%$ & ---- & - & -- & $-=$ & $0,69 \%$ \\
\hline & Nacidos vivos & Nacidos vivos & & 19.87 & & \\
\hline
\end{tabular}


TABLA F

1965

DEFUNCIONES MATERNAS SEGUN INDICE

CODIGO "STANDARD" $7 \times 0$-y01

\begin{tabular}{|c|c|c|c|c|c|}
\hline Historia & Edad & $\begin{array}{c}\text { Causa de la defunción según } \\
\text { historia }\end{array}$ & Historia & ia Edad & $\begin{array}{c}\text { Causa de la defunción según } \\
\text { historia }\end{array}$ \\
\hline 123.205 & 21 & Eclampsia - Hidronefrosis & 139.382 & 25 & Edema agudo del pulmón \\
\hline 129.192 & 25 & Hemorragia intracraneana & 139.538 & 18 & Peritonitis, aborto séptico \\
\hline 129.514 & 21 & Toxemia - insuficiencia card. & 140.145 & 35 & Hemorragia intracraneana, \\
\hline 128.279 & 22 & $\begin{array}{l}\text { Glomerulonefritis - uremia - } \\
\text { toxemia }\end{array}$ & 130.780 & 26 & $\begin{array}{l}\text { toxemia } \\
\text { Nefrosis - toxemia }\end{array}$ \\
\hline 130.705 & 38 & Insuficiencia renal aguda & 139.532 & 38 & Accidente cardiovascular \\
\hline 79.745 & 25 & Peritonitis generalizada & 140.726 & 35 & Toxemia \\
\hline 131.515 & 37 & Ruptura hepática & 140.725 & 25 & Hemorragia intracraneana \\
\hline 130.930 & 30 & Embolia gaseosa & 140.510 & 28 & Sin especificar \\
\hline 129.627 & 24 & Glomerulonefritis - toxemia & \multirow{3}{*}{$\begin{array}{l}140.671 \\
141.075\end{array}$} & 18 & Septicemia, aborto séptico \\
\hline 131.757 & 28 & Peritonitis & & 25 & Hemorragia intracraneana, \\
\hline 113.128 & 21 & Sept. puerperal, Aborto sep. & & & toxemia \\
\hline 133.349 & 19 & Eclampsia & 115.381 & 26 & Aborto provocado, séptico \\
\hline 133.536 & 23 & Oclusión intestinal, peritonitis & \multirow{2}{*}{$\begin{array}{l}133.767 \\
141.635\end{array}$} & 32 & Glomerulonefritis, toxemia \\
\hline 119.790 & 58 & Paro cardíaco quirúrgico & & 30 & Edema agudo del pulmón \\
\hline 116.820 & 35 & Placenta previa & 141.799 & 34 & Toxemia, eclampsia \\
\hline 111.088 & 20 & Nefrosis, bronconeumonía & 142.324 & 42 & Toxemia \\
\hline 136.638 & 40 & Embolia amniótica & 142.712 & 30 & Sin especificar \\
\hline 79.642 & 39 & Pelviperitonitis & 90.444 & 26 & Toxemia, eclampsia \\
\hline 137.451 & 32 & Peritonitis, coma diabético & \multirow{2}{*}{\multicolumn{2}{|c|}{103.470}} & Aborto séptico, septicemia \\
\hline 137.588 & 30 & Bronconeumonía & & & \\
\hline 134.062 & 28 & Glomerulonefritis & \multirow[t]{3}{*}{1964} & Defunciones maternas & maternas \\
\hline 72.509 & 43 & Peritonitis, maniob. abort. & & --- & $-----=0,20 \%$ \\
\hline 90.250 & 36 & Miocarditis & & Partos aten & 17.751 \\
\hline 24.718 & 34 & Gangrena gaseosa & & & \\
\hline 8.371 & 28 & Insuficiencia renal aguda & 1965 & Defunciones & maternas \\
\hline 85.386 & 35 & Insuficiencia cardíaca & & --- & $--=0,23 \%$ \\
\hline 27.197 & 26 & Meningitis tuberculosa & & Partos aten & 19.074 \\
\hline
\end{tabular}


TABLA $F$ bis

MORTALIDAD MATERNA - 1966

\begin{tabular}{|c|c|c|c|c|c|}
\hline Historia & Edad & $\begin{array}{c}\text { Causa de la defunción según } \\
\text { historia }\end{array}$ & Historia & Edad & $\begin{array}{c}\text { Causa de la defunción según } \\
\text { historia }\end{array}$ \\
\hline 132.506 & 22 & $\begin{array}{l}\text { Aborto séptico. Endome- } \\
\text { tritis aguda }\end{array}$ & 103.400 & 28 & $\begin{array}{l}\text { Aborto séptico. Paro } \\
\text { cardíaco }\end{array}$ \\
\hline 142.817 & 22 & $\begin{array}{l}\text { Aborto provocado. Perito- } \\
\text { nitis. Ileo paralítico }\end{array}$ & $\begin{array}{l}151.832 \\
149.436\end{array}$ & $\begin{array}{l}32 \\
25\end{array}$ & $\begin{array}{l}\text { Ruptura uterina. Anemia } \\
\text { Toxemia. Glomerulonefritis }\end{array}$ \\
\hline 134.316 & 20 & $\begin{array}{l}\text { Peritonitis generalizada. } \\
\text { Sepsis }\end{array}$ & $\begin{array}{r}96.971 \\
152.704\end{array}$ & $\begin{array}{l}21 \\
24\end{array}$ & Pancreatitis \\
\hline $\begin{array}{l}144.256 \\
144.635\end{array}$ & $\begin{array}{l}25 \\
20\end{array}$ & $\begin{array}{l}\text { Peritonitis. Sepsis } \\
\text { Hemorragia cerebral. } \\
\text { Toxemia }\end{array}$ & 152.853 & 29 & $\begin{array}{l}\text { Toxemia } \\
\text { Aborto séptico. Embolia } \\
\text { pulmonar }\end{array}$ \\
\hline $\begin{array}{r}113.178 \\
55.703\end{array}$ & $\begin{array}{l}25 \\
35\end{array}$ & $\begin{array}{l}\text { Sin dato } \\
\text { Tumor cerebral }\end{array}$ & 153.098 & 38 & $\begin{array}{l}\text { Aborto incompleto. Infar- } \\
\text { to pulmonar }\end{array}$ \\
\hline 104.703 & 23 & $\begin{array}{l}\text { Aborto provocado. Peritoni- } \\
\text { tis generalizada. Sepsis }\end{array}$ & 142.371 & 27 & $\begin{array}{l}\text { Peritonitis aguda. Sepsis. } \\
\text { Apendicitis aguda }\end{array}$ \\
\hline $\begin{array}{l}62.514 \\
86.713\end{array}$ & $\begin{array}{l}26 \\
29\end{array}$ & $\begin{array}{l}\text { Peritonitis generalizada } \\
\text { Bronconeumonía }\end{array}$ & 152.855 & 40 & $\begin{array}{l}\text { Parto con cesárea. Infec- } \\
\text { ción amniótica }\end{array}$ \\
\hline 143.666 & 28 & $\begin{array}{l}\text { Peritonitis. Septicemia. } \\
\text { Insuficiencia hepática }\end{array}$ & 153.411 & 17 & $\begin{array}{l}\text { Hemorragia intracraneana. } \\
\text { Eclampsia, toxemia. }\end{array}$ \\
\hline 145.772 & 32 & Peritonitis aguda & 150.662 & 17 & Edema agudo del pulmón \\
\hline 146.048 & 40 & $\begin{array}{l}\text { Peritonitis. Insuficiencia } \\
\text { cardíaca } \\
\text { Gangrena }\end{array}$ & $\begin{array}{r}12 . \\
153 .\end{array}$ & $\begin{array}{l}30 \\
19\end{array}$ & $\begin{array}{l}\text { Aborto séptico. Septicemia } \\
\text { Insuficiencia renal. Glome- } \\
\text { rulonefritis. Toxemia }\end{array}$ \\
\hline 140.906 & 25 & $\begin{array}{l}\text { Edema y congestión cerebral. } \\
\text { (Parto fuera del } \mathrm{S} \text { ) }\end{array}$ & $\begin{array}{l}153.857 \\
140.224\end{array}$ & $\begin{array}{l}28 \\
28\end{array}$ & $\begin{array}{l}\text { Aborto séptico. Septicemia } \\
\text { Aborto provocado. He- }\end{array}$ \\
\hline 146.463 & 19 & Maniobras abortivas. Sepsis & & & patitis. Sepsis \\
\hline 145.947 & ? & $\begin{array}{l}\text { Toxemia. Hemorragia } \\
\text { intracraneana }\end{array}$ & $\begin{array}{l}154.084 \\
154.098\end{array}$ & $\begin{array}{l}18 \\
19\end{array}$ & $\begin{array}{l}\text { Aborto séptico. Peritonitis } \\
\text { Hemorragia intracraneana. }\end{array}$ \\
\hline 130.245 & 41 & $\begin{array}{l}\text { Aborto séptico. } \\
\text { Endometritis aguda }\end{array}$ & 15 & 23 & $\begin{array}{l}\text { Eclampsia. Toxemia } \\
\text { Insuficiencia cardíaca }\end{array}$ \\
\hline 147.417 & 31 & Colagenosis & 153 & 36 & Accidente cardiovascular \\
\hline 108.996 & 28 & Edema cerebral. Toxemia & 154.334 & 31 & Eclampsia. Toxemia \\
\hline $\begin{array}{l}147.535 \\
147.880\end{array}$ & $\begin{array}{l}16 \\
27\end{array}$ & $\begin{array}{l}\text { Hemorragia intracraneana } \\
\text { Eclampsia. Toxemia }\end{array}$ & 154.728 & 17 & $\begin{array}{l}\text { Aborto séptico. Peritonitis } \\
\text { Septicemia }\end{array}$ \\
\hline $\begin{array}{r}59.830 \\
147.950\end{array}$ & $\begin{array}{l}27 \\
41 \\
18\end{array}$ & $\begin{array}{l}\text { Embolia pulmonar } \\
\text { Paro respiratorio }\end{array}$ & 3.951 & 32 & $\begin{array}{l}\text { Aborto séptico. Insuficien- } \\
\text { cia renal }\end{array}$ \\
\hline 147.167 & 23 & $\begin{array}{l}\text { Aborto provocado. Septicemia. } \\
\text { Pelviperitonitis }\end{array}$ & $\begin{array}{l}41.012 \\
83.215\end{array}$ & $\begin{array}{l}33 \\
37\end{array}$ & $\begin{array}{l}\text { Piohemia } \\
\text { Hemorragia interna. Des- }\end{array}$ \\
\hline 46.619 & 34 & $\begin{array}{l}\text { Aborto incompleto. } \\
\text { Embolia pulmonar }\end{array}$ & 15 & 27 & $\begin{array}{l}\text { prendimiento de placenta } \\
\text { Leucemia }\end{array}$ \\
\hline 147.874 & 23 & $\begin{array}{l}\text { Aborto séptico. Insufi- } \\
\text { ciencia renal } \\
\text { Bronconeumonía }\end{array}$ & $\begin{array}{l}108.419 \\
155.485\end{array}$ & $\begin{array}{l}42 \\
28\end{array}$ & $\begin{array}{l}\text { Septicemia } \\
\text { Aborto provocado. } \\
\text { Septicemia }\end{array}$ \\
\hline 148.297 & 26 & $\begin{array}{l}\text { Aborto séptico } \\
\text { Insuficiencia renal }\end{array}$ & $\begin{array}{l}132.980 \\
156.041\end{array}$ & $\begin{array}{l}33 \\
27\end{array}$ & $\begin{array}{l}\text { Gangrena gaseosa } \\
\text { Ruptura hepática }\end{array}$ \\
\hline $\begin{array}{l}148.968 \\
149.302\end{array}$ & $\begin{array}{r}23 \\
?\end{array}$ & $\begin{array}{l}\text { Pertionitis. Sepsis } \\
\text { Infarto del miocardio }\end{array}$ & 141.218 & 38 & $\begin{array}{l}\text { Aborto séptico. Insuficiencia } \\
\text { renal }\end{array}$ \\
\hline 148.874 & 24 & Insuficeincia renal. Sepsis & 157.221 & $?$ & Eclampsia \\
\hline 148.943 & 17 & $\begin{array}{l}\text { Peritonitis. Sepsis. Ruptura } \\
\text { uterina. Edema pulmonar }\end{array}$ & 7.743 & 38 & $\begin{array}{l}\text { Aborto incompleto. Glome- } \\
\text { rulonefritis. Edema pulm. }\end{array}$ \\
\hline $\begin{array}{l}149.375 \\
149.166\end{array}$ & $\begin{array}{l}29 \\
28\end{array}$ & $\begin{array}{l}\text { Gangrena gaseosa. Sepsis } \\
\text { Septicemia }\end{array}$ & $\begin{array}{r}66.101 \\
157.509\end{array}$ & $\begin{array}{l}28 \\
20\end{array}$ & $\begin{array}{l}\text { Aborto séptico. Peritonitis } \\
\text { Eclampsia }\end{array}$ \\
\hline 127.384 & 28 & Septicemia & 158.417 & 38 & Aborto séptico. Tétanos \\
\hline 105.362 & 33 & $\begin{array}{l}\text { Paro cardíaco. Edema } \\
\text { agudo del pulmón } \\
\text { Aborto séptico. }\end{array}$ & $\begin{array}{l}115.727 \\
158.126\end{array}$ & $\begin{array}{l}35 \\
42\end{array}$ & $\begin{array}{l}\text { Ruptura hepática. Toxemia } \\
\text { Hemorragia intracraneana. } \\
\text { Eclampsia }\end{array}$ \\
\hline 150.725 & 22 & $\begin{array}{l}\text { Aborto séptico. } \\
\text { Insuficiencia renal }\end{array}$ & \multirow{3}{*}{\multicolumn{2}{|c|}{$\begin{array}{l}\text { Total de defun } \\
\text { maternas }\end{array}$}} & $\begin{array}{l}\text { Eclampsia } \\
\text { iones }\end{array}$ \\
\hline $\begin{array}{l}150.181 \\
151.067\end{array}$ & $\begin{array}{l}24 \\
34\end{array}$ & $\begin{array}{l}\text { Edema pulmonar } \\
\text { Septicemia. Bronconeumonía. }\end{array}$ & & & $77 \%$ sobre \\
\hline 151.067 & 34 & $\begin{array}{l}\text { Septicemia. Bronconeumonía. } \\
\text { Aborto séptico. Peritonitis }\end{array}$ & & & 20.326 \\
\hline
\end{tabular}


TABLA G

\author{
INDICES BIOGRAFICCS Y DOCENTES DEL INSTITUTO MATERNO INFANTIL \\ "CONCEPCION VILLAVECES DE ACOSTA" EN 1.965
}

\begin{tabular}{lr}
\hline & Promedio anual \% \\
\hline Coeficiente de inmadurez sobre nacidos vivos & 0,63 \\
Coeficiente de prematurez sobre nacidos vivos & 15,40 \\
Coeficiente de embarazos a término: A) sobre nacidos vivos & 0,48 \\
B) sobre partos a término con feto vivo t a término con feto muerto & 88,02 \\
Coeficiente de abortos sobre embarazos terminados & 27,99 \\
Coeficiente de abortos sobre partos atendidos & 21,76 \\
Coeficiente de abortos sobre nacidos vivos & 24,61 \\
Coeficiente de mortinatalidad sobre embarazos terminados & 3,38 \\
Coeficiente de mortinatalidad sobre partos atendidos & 2,92 \\
Coeficiente de mortinatalidad sobre nacidos vivos & 2,96 \\
Coeficiente de desperdicio demográfico antenatal & 26,76 \\
Coeficiente de cesárea sobre total de partos atendidos & 3,99 \\
Coeficiente de ventosa sobre total de partos atendidos & 0,25 \\
Coeficiente de versión interna sobre total de partos atendidos & 0,087 \\
Coeficiente de E. podálica sobre total de partos atendidos & 0,083 \\
Coeficiente de parto intervenido en general sobre total de partos atendidos & 9,60 \\
Coeficiente de fórceps sobre total de partos atendidos & 5,07 \\
Coeficiente de espátulas sobre total de partos atendidos & 0,14 \\
Coeficiente de parto intervenido por maniobras & 0,44 \\
Coeficiente de mortalidad materna sobre partos atendidos & 0,23 \\
\hline
\end{tabular}

TABLA $G$ bis

INDICES BIOGRAFICOS $Y$ DOCENTES DEL INSTITUTO MATERNO INFANTIL "CONCEPCION VILLAVECES DE ACOSTA" EN 1.966

\begin{tabular}{lr}
\hline & Promeclio anual \% \\
\hline Coeficiente de inmadurez sobre nacidos vivos & 0,85 \\
Coeficiente de prematurez sobre nacidos vivos & 15,06 \\
Coeficiente de embarazos a término: A) sobre nacidos vivos & 0,48 \\
B) sobre partos a término con feto vivo + a término feto muerto & 86,26 \\
Coeficiente de abortos sobre embarazos terminados & 31,26 \\
Coeficiente de abortos sobre partos atendidos & 26,03 \\
Coeficiente de abortos sobre nacidos vivos & 26,89 \\
Coeficiente de mortinatalidad sobre embarazos terminados & 3,75 \\
Coeficiente de mortinatalidad sobre partos atendidos & 3,16 \\
Coeficiente de mortinatalidad sobre nacidos vivos & 3,27 \\
Coeficiente de desperdicio demográfico antenatal & 28,10 \\
Coeficiente de cesárea sobre total de partos atendidos & 4,62 \\
Coeficiente de ventosa sobre total de partos atendidos & 0,25 \\
Coeficiente de versión interna sobre total de partos atendidos & 0,10 \\
Coeficiente de extracción podálica sobre total de partos atendidos & 0,11 \\
Coeficiente de parto intervenido en general sobre total de partos atendidos & 10,67 \\
Coeficiente de fórceps sobre total de partos atendidos & 5,01 \\
Coeficiente de espátulas sobre total de partos atendidos & 0,18 \\
Coeficiente de parto intervenido por maniobras & 0,38 \\
Coeficiente de mortalidad materna sobre total de partos atendidos & 0,37 \\
\hline
\end{tabular}


TABLA H

OBSERVACIONES - 1965

\begin{tabular}{|c|c|c|}
\hline Porcentaje de ocupación de & le camas & $88,12 \%$ \\
\hline Promedio de permanencia & (días) & $4,38 \%$ \\
\hline Mortalidad materna & & $0,23 \%$ \\
\hline Mortalidad infantil & & $2,07 \%$ \\
\hline Toxemias: & & \\
\hline Total de ingresadas & 386 & \\
\hline------- & $--=$ & $20,24 \%$ \\
\hline Partos atendidos & 19.074 & \\
\hline Porcentaje de autopsias: & & \\
\hline Autopsias (adultas) & 45 & \\
\hline------- & $--=$ & $100,00 \%$ \\
\hline Defunciones & 45 & \\
\hline Muertes por inmadurez & 52 & \\
\hline------ & $--=$ & $0,28 \%$ \\
\hline Nacidos vivos & 18.684 & \\
\hline Niños sanos & 15.665 & \\
\hline------ & $--=$ & $83,84 \%$ \\
\hline Nacidos vivos & 18.684 & \\
\hline Niños enfermos & 3.019 & \\
\hline------ & $--=$ & $16,16 \%$ \\
\hline Nacidos vivos & 18.684 & \\
\hline
\end{tabular}

TABLA $\mathrm{H}$ bis

OBSERVACIONES. - RESUMEN

1966

Porcentaje de ocupación de camas:

Estancias $\quad 94.587$

- - - - - - - - = $70,997 \%$

Cupo de camas: $\quad 365 \times 365$

Promedio de permanencia:

Estancias 94.587

- - - - - - - - $=3,33 \mathrm{días}$

Egresos

28.391

Mortalidad materna (porcentajes)

Muertes maternas
$------\frac{77}{20.326}=0,378 \%$
Partos atendidos

Muertes maternas 77

- - - - - - - - $=1,368 \%$

Abortos $\quad 5.555$

Muertes maternas 77

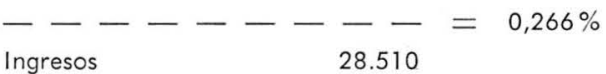

TOXEMIAS (porcentaje):

Ingresadas 397

$--------1,95 \%$

Autopsias maternas (porcentaje)

\begin{tabular}{|c|c|c|c|c|}
\hline Autopsias & & 62 & & \\
\hline----- & - & -- & $=$ & $80,51 \%$ \\
\hline Defunciones & & 77 & & \\
\hline Niños sanos & & 16.488 & & \\
\hline----- & - & -- & $=$ & $82,96 \%$ \\
\hline Nacidos vivos & & 19.874 & & \\
\hline Niños enfermos & & 4.209 & & \\
\hline----- & - & -- & $=$ & $21,17 \%$ \\
\hline Nacidos vivos & & 19.874 & & \\
\hline Defunciones infantiles & ( po & rcentaje) & & \\
\hline Defunciones infantiles & & 638 & & \\
\hline----- & - & -- & $=$ & $3,21 \%$ \\
\hline Nacidos vivos & & 19.874 & & \\
\hline
\end{tabular}


TABLA I

INSTITUTO MATERNO INFANTIL "CONCEPCION VILLAVECES DE ACOSTA"

SERVICIOS PEDIATRICOS - 1965 - (Doctor Alberto Amarís Mora, Jefe)

\begin{tabular}{|c|c|c|c|c|c|c|c|c|c|c|c|}
\hline & $\begin{array}{l}\text { Recién Nac. } \\
\text { Lastantes }\end{array}$ & Lactantes & Hidratación & $\begin{array}{l}\text { Premat. no } \\
\text { infectados }\end{array}$ & $\begin{array}{l}\text { Prematuros } \\
\text { infectados } \\
\text { Instituto }\end{array}$ & $\begin{array}{l}\text { Prematuros } \\
\text { Infectados } \\
\text { de la calle }\end{array}$ & $\begin{array}{l}\text { Prematuros } \\
\text { Recuperación }\end{array}$ & Problemas & $\begin{array}{l}\text { Aislamiento } \\
2 \% \text { piso }\end{array}$ & $\begin{array}{l}\text { Cirugía } \\
\text { general }\end{array}$ & $\begin{array}{l}\text { Cirugía } \\
\text { plástica }\end{array}$ \\
\hline Estancias & 3.116 & 5.938 & 4.669 & 9.566 & 2.516 & 2.928 & 4.700 & 5.356 & 2.059 & 463 & 533 \\
\hline Ingresos & 138 & 688 & 486 & 1.397 & 342 & 379 & 335 & 2.825 & 250 & 46 & 47 \\
\hline Egresados vivos & 106 & 548 & 414 & 1.074 & 195 & 213 & 338 & 2.743 & 229 & 37 & 46 \\
\hline $\begin{array}{l}\text { Estancias egresados vivos, } \\
\text { promedio }\end{array}$ & 10,3 días & 12,2 días & 10 días & 8 días & 11,5 días & 18,5 días & 16,2 días & 1,7 días & 6,5 días & 9,6 días & 11,3 días \\
\hline Defunciones & 42 & 130 & 68 & 307 & 148 & 164 & 4 & 75 & 22 & 11 & 2 \\
\hline Defunciones antes de 24 horas & 16 & 52 & 25 & 113 & 39 & 50 & 1 & 41 & 11 & 5 & 0 \\
\hline $\begin{array}{l}\text { Estancias egresados muertos, } \\
\text { promedio }\end{array}$ & 5,3 días & 8,8 días & 4,9 días & 4,6 días & 5,4 días & 4,6 días & 0,5 días & 2,1 días & 2,8 días & 4 días & 1,7 días \\
\hline Mortalidad sobre egresos & $28,3 \%$ & $19,17 \%$ & $14,1 \%$ & $22,2 \%$ & $43,1 \%$ & $43,5 \%$ & $1,1 \%$ & $2,6 \%$ & $8,7 \%$ & $22,9 \%$ & $4,1 \%$ \\
\hline Indice de ocupación & $72 \%$ & $107,9 \%$ & $93,9 \%$ & $65,6 \%$ & $39,7 \%$ & $46,1 \%$ & $61,1 \%$ & $63,4 \%$ & $56,3 \%$ & $37,2 \%$ & $49,9 \%$ \\
\hline $\begin{array}{l}\text { Consultas de primera vez } \\
\text { Consultas diurnas }\end{array}$ & & & $\begin{array}{r}7.542 \\
26.701\end{array}$ & CONSI & A EXTERNA & \multicolumn{3}{|c|}{$\begin{array}{l}\text { Consultas nocturnas } \\
\text { Total de consultas }\end{array}$} & & & $\begin{array}{r}3.441 \\
30.142\end{array}$ \\
\hline
\end{tabular}


TABLA I bis

INSTITUTO MATERNO INFANTIL "CONCEPCION VILLAVECES DE ACOSTA" SERVICIOS PEDIATRICOS - 1965 - (Doctor Alberto Amarís Mora, Jefe)

\begin{tabular}{|c|c|c|c|c|c|c|c|c|c|c|}
\hline & Lastantes & Hidratación & $\begin{array}{l}\text { Premat. no } \\
\text { infectados }\end{array}$ & $\begin{array}{l}\text { Prematuros } \\
\text { infectados } \\
\text { Instituto }\end{array}$ & $\begin{array}{l}\text { Prematuros } \\
\text { Infectados } \\
\text { de la calle }\end{array}$ & $\begin{array}{l}\text { Prematuros } \\
\text { Recuperación }\end{array}$ & Problemas & $\begin{array}{l}\text { Aislamiento } \\
2: \text { piso }\end{array}$ & $\begin{array}{l}\text { Cirugía } \\
\text { general }\end{array}$ & $\begin{array}{l}\text { Cirugía } \\
\text { plástica }\end{array}$ \\
\hline Ingresos & 1.049 & 689 & 1.054 & 429 & 455 & 451 & 3.265 & 285 & 71 & 49 \\
\hline Estancias & 9.191 & 4.696 & 11.827 & 2.908 & 3.778 & 5.172 & 6.775 & 2.661 & 994 & 532 \\
\hline Egresados vivos & 908 & 619 & 1.194 & 231 & 273 & 437 & 3.203 & 251 & 54 & 46 \\
\hline Defunciones & 143 & 65 & 287 & 135 & 184 & 3 & 62 & 34 & 15 & 2 \\
\hline Defunciones antes de 24 horas & 56 & 20 & 111 & 20 & 82 & 0 & 36 & 10 & 4 & 0 \\
\hline $\begin{array}{c}\text { Promedio estancias } \\
\text { egresados vivos }\end{array}$ & 14 días & 7 días & 7 días & 11 días & 12 días & 10 días & 2 días & 12 días & 15 días & 9 días \\
\hline $\begin{array}{l}\text { Promedio estancias } \\
\text { egresados muertos }\end{array}$ & 11 días & 7 días & 3 días & 6 días & 7 días & 1 día & 1 día & 9 días & 7 días & 0,5 días \\
\hline Mortalidad sobre egresos & $26 \%$ & $9 \%$ & $19 \%$ & $37 \%$ & $39 \%$ & $0,6 \%$ & $2 \%$ & $13 \%$ & $22 \%$ & $4 \%$ \\
\hline Coeficiente de ocupación & $87 \%$ & $91 \%$ & $64 \%$ & $54 \%$ & $68 \%$ & $71 \%$ & $81 \%$ & $73 \%$ & $45 \%$ & $29 \%$ \\
\hline \multicolumn{11}{|c|}{ CONSULTA EXTERNA } \\
\hline $\begin{array}{l}\text { Consultas de primera vez } \\
\text { Consultas diurnas }\end{array}$ & \multicolumn{4}{|c|}{$\begin{array}{r}8.252 \\
29.212\end{array}$} & \multicolumn{3}{|c|}{$\begin{array}{l}\text { Consultas nocturnas } \\
\text { Total de corisultas }\end{array}$} & & & $\begin{array}{r}3.967 \\
33.179\end{array}$ \\
\hline
\end{tabular}




\title{
BIOPSIA RENAL PERCUTANEA:
}

\section{INDICACIONES, CONTRAINDICACIONES Y COMPLICACIONES*}

\author{
Dr. Hernando Rocha Posada**
}

Varias nuevas entidades patológicas y clínicas se han descrito desde la introducción en la práctica médica de la biopsia renal. Ha sido posible descle entonces precisar y detectar cambios no vistos en el material post-mortem, en el cual, como es sabido, los tejidos son afectados por la autólisis, permitiendo solo estimar el curso vascular y algunos cambios inflamatorios en el tejido conectivo.

La práctica de este procedimiento en los pacientes con enfermedad renal se impone toda vez que se desee us diagnóstico correcto mientras no se ponga en peligro la vida del individuo. Su estudio es de utilidad principalmente en las afecciones difusas $(29,30,37,40)$ y en menor grado en ciertas lesiones, habitualmente locales, como pueden ser la pielonefritis, los tumores, y las inflamaciones específicas (40). Aporta la seguridad necesaria, no obtenida por los métodos clínicos, tanto en el diagnóstico como en el pronóstico de numerosas enfermedades $(1,8,9,12,13,24$, $28,29,30,40,46,48)$, además de permitir establecer una terapéutica adecuada y valorar sus resultados $(28,29,40)$.

Desde el punto de vista investigativo, los hallazgos clínicos y de labo- ratorio, especialmente funcionales, son hoy día correlacionados con las anormalidades histológicas (26, 36). Así mediante biopsias seriadas ha sido posible conocer la evolución de las enfermedades, hecho de capital importancia en la comprensión de la historia natural de las afecciones renales y cardiovasculares (37). Ha permitido dilucidar el problema de las nefropatías unilaterales, ciertos aspectos de fisiología, fisiopatología, citoquímica $(14,30,44)$, citodiagnóstico y las alteraciones estructurales producidas por medicamentos (14). Finalmente, se la ha utilizado en inmuno-patología (3), así como para el cultivo de células renales y del mismo tejido renal $(23,28,46$, 49).

El desarrollo de un método seguro para obtener material mediante biopsia percutánea, permite hoy día conocer y seguir el curso de ciertas enfermedades que complican el embarazo. Cada afección vasculo-renal de la gestación tiene una lesión característica. En la pre-eclampsia por

* Trabajo realizado en las Unidades de Patología Infecciosa y Obstetricia de la Fac. de Med. de la Universidad Nal.

** Profesor Asistente de Medicina Interna. Director de la Unidad de Patología Infecciosa. Hosp. San Juan de Dios. Bogotá. 
ejemplo, se demuestra siempre una lesión específica y diagnóstica (10), no hallada en los embarazos normales o en los casos de enfermedad hipertensiva sin toxemia.

A través de los años se ha comprendido de manera lógica una complicación de extremada frecuencia en nuestro medio y que hasta hace po$\mathrm{co}$, en todas partes del mundo, era diagnosticada y manejada con métodos exclusivamente clínicos y de laboratorio. Me refiero a la toxemia del embarazo. En esta entidad, con la biopsia renal el diagnóstico es certero y el pronóstico posible, tanto para la madre como para el feto, estableciéndose el grado de lesiones anatómicas y aclarando, en algunos casos, enfermedades previas al embarazo o su agravación por el mismo (20).

La tendencia corriente a clasificar a toda embarazada que ha tenido o tiene convulsiones de etiología oscura como eclámptica, merece revisarse. El diagnóstico clínico de gestosis hipertensiva genuina, basado únicamente en la sintomatología y en los exámenes de laboratorio de rutina, complementados con el funcionalismo renal, es incierto y sujeto a errores acerca de la verdadera índole de la lesión parenquimatosa y del porvenir alejado de la paciente. Desde 1877, año en que Bertles (cit. 20) se interesó en el riñón de las eclámpticas, todos los hallazgos fueron de autopsia, hasta que Dickman (11) en 1940 practicó por primera vez biopsias del riñón durante la cesárea. El aporte hecho por Dickman es de suma importancia ya que todos sabemos que los estudios anatomo-patológicos, sobre material de autopsia, solo muestran los casos terminales y en éstos no se puede afirmar que las lesiones descubiertas sean debidas a una toxe- mia que ocurrió muchas décadas antes (59).

Hipertensión arterial, proteinuria y edema son los signos cardinales del síndrome toxémico. Debido a que algunos o todos pueden ocurrir en embarazos complicados con enfermedad vascular hipertensiva, enfermedad renal pura o la combinación de las dos, el diagnóstico seguro de su causa se impone. Schewitz y col. (52), estudiando un grupo de 77 embarazadas con síndrome toxémico, hallaron solo 9 casos $(11,6 \%)$ con lesiones propias de pre-eclampsia-eclampsia, y una alta incidencia de lesiones asociadas al mismo (principalmente esclerosis arteriolar, nefroesclerosis arteriolar y pielonefritis crónica). Los riesgos de la biopsia renal percutánea, llevada a cabo durante el período evolutivo de la enfermedad, están pues justificados.

Pacientes incluídas en el estudio. Fueron sometidas a este procedimiento 159 hospitalizadas en el Servicio de Toxemias del Instituto Materno Infantil de Bogotá, a donde concurrieron por presentar anormalidades en el curso de su embarazo y/o en el puerperio inmediato (Cuadros Nos. 1, 2, 3).

Las complicaciones observadas durante la primera y segunda mitad del embarazo, así como durante el puerperio inmediato, se han comparado con las que presentó un grupo de 61 pacientes no embarazadas y 39 hombres hospitalizados en la Sección de Medicina Interna del Hospital San Juan de Dios de Bogotá (Cuadro Ni $4)$.

Complicaciones. Durante la primera mitad del embarazo fueron biopsiadas 22 pacientes (Cuadro № 1 ) de las cuales 9 presentaron complicaciones $(40.8 \%)$, de carácter hemorrági- 
CUADRO No 1

PRIMERA MITAD DEL EMBARAZO

\begin{tabular}{lrc}
\hline Enfermedad & No $^{\mathbf{0}}$ & Complicaciones \\
\hline Mola hidatif $(+)$ & 12 & 5 \\
Toxemia & 4 & 1 \\
Pielon. cron. & 2 & 1 \\
Polihidramnios & 1 & 1 \\
Hipert. maligna & 1 & - \\
Amenaza aborto & 1 & 1 \\
Embarazo normal & 1 & - \\
TOTAL & -22 & 9 \\
\hline
\end{tabular}

(t) Con cambios de toxemia en 6 de ellas.

co leve. Estas hematurias fueron siempre macroscópicas y se prolongaron entre 24 y 96 horas sin precisar de transfusión. Un caso presentó conjuntamente cólico renal que cedió con antiespasmódicos y otro un colapso cardiovascular inmediato a la biopsia que cedió espontáneamente.

\section{CUADRO NN 2}

\section{SEGUNDA MITAD DEL EMBARAZO}

\begin{tabular}{|c|c|c|}
\hline Enfermedad & $\mathbf{N}^{o}$ & Complicaciones \\
\hline Toxemia & 68 & 34 \\
\hline Pielonefritis crónica & 4 & 3 \\
\hline Glomérulonefritis crónica & 3 & 1 \\
\hline Síndrome nefrótico & 1 & - \\
\hline \multirow[t]{2}{*}{ Riñón normal } & 1 & 1 \\
\hline & 一 & - \\
\hline TOTAL & 77 & $39(50.6 \%)$ \\
\hline
\end{tabular}

Durante la segunda mitad se estudiaron 77 casos (Cuadro $N:$ 2) ascendiendo el número de complicaciones al 50.6\% (39 casos). La mayoría fueron de tipo hemorrágico, en algunos casos más serias tanto en intensidad como en duración que en el grupo anterior. Así se observó hematuria macroscópica simple, prolongada entre 24 y96 horas en 29 casos $(74,3 \%)$ que tampoco precisaron de ningún tratamiento especial. En un caso $(2,5 \%)$ se observó hemorragia muy severa iniciada a làs cuatro ho- ras de la punción, en la cual fue necesario transfundir $500 \mathrm{cc}$. de sangre cediendo el cuadro en 12 horas; en cuatro $(10,2 \%)$ la hematuria se prolongó por seis días, precisando solo una de ellas de transfusión. Hemorragias mucho más prolongadas se presentaron en dos casos $(5,1 \%)$, de los cuales en una duró 15 y en otra 40 días, siendo necesarias dos y cinco transfusiones de $500 \mathrm{cc}$. de sangre respectivamente. Es interesante anotar que en la primera de ellas la hemorragia se inició tardíamente, al cuarto día de la biopsia. Una paciente con pre-eclampsia presentó un colapso cardiovascular inmediato a la punción, el cual cedió espontáneamente $(2,5 \%)$; sin embargo, 24 horas después de ocurrido, había muerte fetal. El examen de la placenta mostró huellas de desprendimiento en cerca de $60-70 \%$ de su superficie, situación ésta que muy posiblemente contribuyó en buena parte al accidente. Finalmente, en un caso se presentó un hematoma perirrenal con cólico violento y en otro cólico aislado simple que cedieron a la administración de antiespasmódicos.

Durante el puerperio inmediato (Cuadro № 3 ) el número de complicaciones fue todavía superior al observado en la segunda mitad del embarazo. En este período fueron sometidas al procedimiento de biopsia 60 pacientes, de las cuales $37(61,6 \%)$ presentaron complicaciones. En 29 casos $(78,3 \%)$ se observó hematuria macroscópica simple prolongada entre 24 y 72 horas; en cuatro pacientes $(10,8 \%)$ se prolongó por 10 días sin que haya sido necesario trasfundir sangre y en otras dos $(5,4 \%$ ) la hemorragia fue masiva, con abundantes coágulos en la vejiga. Estos dos últimos accidentes fueron inmediatos a la punción y en uno se acompañó de estado de shock, cediendo las dos 
CUADRO NN 3

PUERPERIO INMEDIATO

\begin{tabular}{|c|c|c|}
\hline Enfermedad & $\mathbf{N}^{0}$ & Complicaciones \\
\hline Toxemia $(+)$ & 39 & 23 \\
\hline Mola hidatiforme $(++)$ & 10 & 4 \\
\hline $\begin{array}{l}\text { Pielonefritis crónica } \\
\text { Enf. Vasc. crón. }\end{array}$ & 5 & 5 \\
\hline Hipertensión sin toxemia & 3 & 3 \\
\hline Glomérulonefritis crónica & 2 & 1 \\
\hline \multicolumn{3}{|l|}{ Glomérulonefritis } \\
\hline intercapilar & 1 & 1 \\
\hline & - & - \\
\hline TOTAL & 60 & $37(61.6 \%)$ \\
\hline
\end{tabular}

$(+)$ Asociada a: cambios vasculares de hipertensión en 11 casos, a Pielonefritis crónica en 4 y a Necrosis tubular en dos.

$(++)$ Con cambios de toxemia en 6 .

luego de transfusión de sangre. Finalmente dos casos $(5,4 \%)$ tuvieron como complicación un hematoma perirenal infectado y otro $(2,6 \%)$ una septicemia, cediendo las mencionadas complicaciones luego de tratamiento antibiótico.

En total, sobre las 159 pacientes sometidas a la biopsia, 86 (54,08\%) presentaron complicaciones. Fue la hemorragia macroscópica simple leve la de mayor incidencia $(77,8 \%)$ y solo el $15,1 \%$ la presentaron en forma alarmante 0 muy prolongada, siendo necesario recurri a la transfusión de sangre total en algunos casos. Otras complicaciones como el cólico renal, colapso cardiovascular, shock, muerte fetal, hematoma perirenal infectado o sepsis fueron muy poco frecuentes.

En el grupo de varones y mujeres no embarazadas (Cuadro $\mathrm{N}$ ? 4) solo un $25 \%$ presentaron complicaciones (25 de 100 pacientes estudiados). También fue la hemorragia macroscópica simple leve la más frecuente $(76 \%)$. En dos $(8 \%)$ se prolongó entre 14 y 20 días precisándose en el último de nefrectomía. En tres pacientes $(12 \%)$ se observó cólico re-
Rev. Col. Obst. y Ginec.

\section{CUADRO № 4}

VARONES $Y$ MUJERES NO EMBARAZADAS

\begin{tabular}{lcc}
\hline Enfermedad & No & Complicaciones \\
\hline Pielonefritis crónica & 37 & 12 \\
Glomérulonefritis crónica & 19 & 1 \\
Riñón normal & 18 & 5 \\
Glomérulonefritis subaguda & $5(++)$ & - \\
Nefroesclerosis maligna & 4 & 2 \\
Glomérulonefritis & 4 & - \\
$\quad$ membranosa & 3 & - \\
Nefroarterioloesclerosis & 2 & 2 \\
Glom. difusa aguda & 2 & 1 \\
Hipertensión esencial & 2 & - \\
Gloméruloesclerosis & 2 & 1 \\
intercapilar & 1 & - \\
Síndrome nefrótico & 1 & 1 \\
Escleroderma & - & - \\
Dermatomiositis & 100 & 25 \\
\end{tabular}

(+) Fué necesaria sutura renal por hemorragia.

$(++)$ En un caso fué necesaria la nefrectomía por hemorragia.

nal que cedió con antiespasmódicos $y$ en uno $(4 \%)$ se presentó hematuria muy severa durante 48 horas siendo necesario practicar sutura renal cediendo el cuadro. De dos casos quirúagicos, uno de los cuales padecía una hipertensión maligna, el riñón era de aspecto normal, sin desgarro alguno, con una puntura en su centro; una sutura simple fue suficiente. En el otro paciente, que sufría de una glomérulonefritis sub-aguda, se halló el riñón completamente infartado para lo cual fue necesario la nefrectomía.

\section{Comentarios}

Es la toxemia del embarazo la principal indicación de la biopsia renal durante el ciclo grávido-puerperal. Su práctica permite comprobar una presunción clínica $\mathrm{y} / \mathrm{o}$ de laboratorio, a la vez que hacer el diagnóstico diferencial con las enfermedades que pueden dar lugar a la aparición del síndrome toxémico $(10,11,13,29$, 
47, 48, 52, 59). También es de utilidad en el estudio de ciertas anurias puerperales (53) y de aquellas consecutivas a daño útero-placentario (21).

Si bien éstas pueden ser las indicaciones electivas de la biopsia durante la gravidez o el puerperio, no son las únicas ya que puede requerirse en cualesquiera de los múltiples procesos patológicos que afectan a la no embarazada, bien porque se presentaron antes de la gestación, se agravaron por la misma o aparecieron en su curso. Deben considerarse dentro de este grupo de indicaciones principalmente al síndrome nefrótico $(3,4,7,9,12,13,25,26,30,32$, $37,39,40,57)$, las albuminurias y hematurias renales de etiología incierta $(13,28,29,30,34,37,45)$, algunas de las enfermedades del colágeno $(12,13,15,29,30,36,38,49)$, glomérulonefritis y pielonefritis (5, $9,12,13,23,29,30,40,49)$, hipertensión de causa desconocida $(8,13$, $28,29,37,56)$, nefropatía gotosa y oxalosis (13), cistinosis (22), nefropatía diabética $(5,29,31,49)$, amiloidosis $(13,28,29,49)$, sarcoidosis (19) hiperaldosteronismo secundario $(29,35)$ y anurias consecutivas al estado de shock e intoxicación medicamentosa.

En la actualidad son muy numerosas las contraindicaciones, en comparación con las relatadas cuando se introdujo la biopsia en la práctica clínica. Algunas de ellas no imponen la abstención del precedimiento, sino cuidados especiales en la técnica y manejo posterior del paciente. La mayoría de los investigadores están de acuerdo en considerar como contraindicaciones absolutas a la falta de colaboración del paciente $(9,13$, $17,27,28,29,40)$, diátesis hemorrágica $(9,12,13,17,27,28,29,30$, $34,40,42,45,55)$, riñón único $(9$,
$13,17,28,30,34,37,40,42,45)$, enfermedad renal terminal (13, 17, 38), hidronefrosis e hidropionefrosis $(13,17,28,29,34,40,41,53,58)$, perinefritis supurada y grandes quistes $(17,27,28,40,45)$, aneurismas de la arteria renal $(9,17,27,28$, $41)$, arterioesclerosis con calcificaciones arteriales extensas $(17,18,29$, 40, 42), hipovolemia (12, 13, 18, 42), fístula arteriovenosa renal (13), inexperiencia del operador y curiosidad académica (42). White (42), la contraindica en la insuficiencia renal con oliguria aguda y aumento rápido y progresivo de los niveles de úrea. Finalmente, las neoplasias y la tuberculosis son consideradas como contraindicaciones para el procedimiento por algunos investigadores (13, $17,28,34,40,41)$. Sin embargo Miatello y col. (37) no hallan diferencia entre las tomas hechas con pinzas sacabocados y con electrocoagulación y el procedimiento en este estudio empleado; practicaron biopsias en 15 neoplasias y 18 tuberculosis sin diseminación alguna.

Figuran entre las contraindicaciones relativas $(9,13,30,34,41,42$, $45,55)$, la hipertensión arterial con mínima sostenida por encima de 110 $\mathrm{mm}$. de mercurio, insuficiencia cardíaca congestiva, obesidad severa e hiperazohemia rápidamente progresiva (superior a $100 \mathrm{mg}$. por $100 \mathrm{~cm}^{3}$ ). Esta hiperazohemia, (12, 17, 28, 29, $34,37,40,41,42)$, rara vez relativa como contraindicación, es en la experiencia de Brun (6), condicionante de un mayor riesgo de hemorragia. Experimentalmente en perros, Streicher y Bollman y Mann (cit. 60), produjeron hemorragias generalizadas luego de inyectarles úrea y abocar los uréteres al intestino.

La hemorragia y el daño de otra estructura son las complicaciones más comunes. La opinión es que todos los 
pacientes presentan hematuria microscópica $(1,2,9,19,21,27,30$, $33,40,41,42,45,51)$. En una buena proporción de pacientes la hematuria es macroscópica y leve, generalmente precoz aunque puede ser tardía $(31,51)$ y/o prolongarse por varios días $(1,51)$. Numerosos autores $(12,27,28,37,40,41,58)$ relatan hematurias serias entre 0.8 a $13 \%$ de los casos que generalmente no requieren de transfusión y entre 0,1 y $8,8 \%$ que sí la precisan. En el grupo de embarazadas y puérperas observadas, un $4,4 \%$ tuvieron hemorragia que necesitaron de transfusión, e ncomparación con el $2.0 \%$ del grupo de varones y no embarazadas. Esta complicación se halla por lo tanto dentro de las posibilidades relatadas por otros grupos (Cuadro No 7). La hemorragia macroscópica simple leve, también fue más frecuente en el grupo de embarazadas que en el de no embarazadas. Es posible que ciertos factores como la gestación renal durante el embarazo normal y la disminución del fibrinógeno, de la resistencia parietal vascular, la hipertensión e hiperazohemia, en el complicado con toxemia, intervengan en su aparición. En el presente estudio se ha podido observar que el porcentaje de hematurias en pacientes con hipertensión y con tensión normal es similar (Cuadro № 5). Se halló sí al- guna diferencia, en lo que a esta complicación se refiere, entre el grupo de pacientes con elevación del nitrógeno ureico y el grupo que lo tenía dentro de límites normales. Sin embargo, ninguno de los pacientes del primer grupo presentaron niveles superiores a $100 \mathrm{mg} . \%$. Débese admitir que los grupos no son comparativos numéricamente y por lo tanto estos datos tienen un valor estadístico relativo (Cuadro No 6).

La hemorragia macroscópica habitualmente no precisa de transfusión, excepto que sea muy profusa o muy prolongada. En la embarazada este tipo de hematuria requiere la atención inmediata, toda vez que ella puede afectar seriamente el feto (2), especialmente si éste se halla en desventaja por toxemia, enfermedad crónica vascular hipertensiva, diabetes, pielonefritis, etc. El shock hemorrágico es siempre una complicación grave que requiere de atención efectiva e inmediata como manera de salvar a la paciente y a su producto $(9,12$, 58). Complicaciones igualmente serias con el hematoma subcapsular (9, $12,21,28,30,40,42,44,51,54)$, el hematoma retroperitoneal masivo $(29,40)$ y el infarto renal (29). Tales complicaciones se deben generalmente a la mala fijación del riñón durante el acto de la biopsia y casi

CUADRO N: 5

COMPLICACION HEMORRAGICA - CORRELACION CON LOS NIVELES TENSIONALES

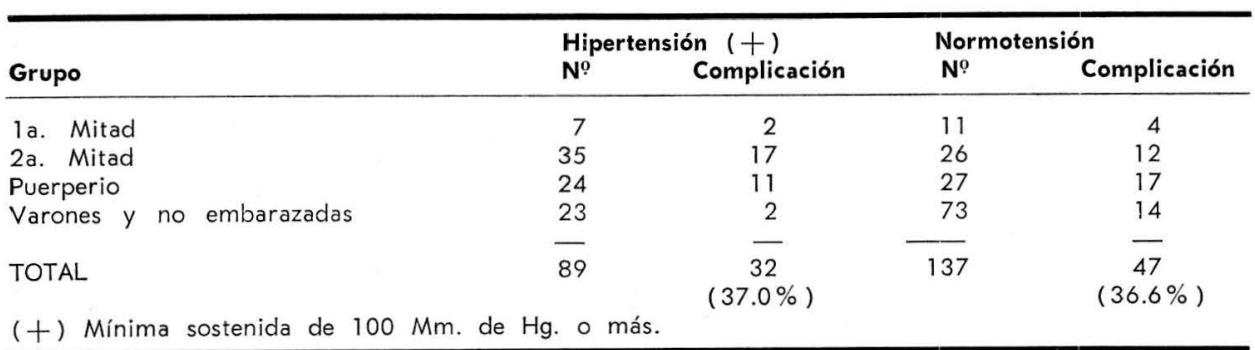


CUADRO N: 6

COMPLICACION HEMORRAGICA

CORRELACION CON LOS NIVELES DE NITROGENO UREICO

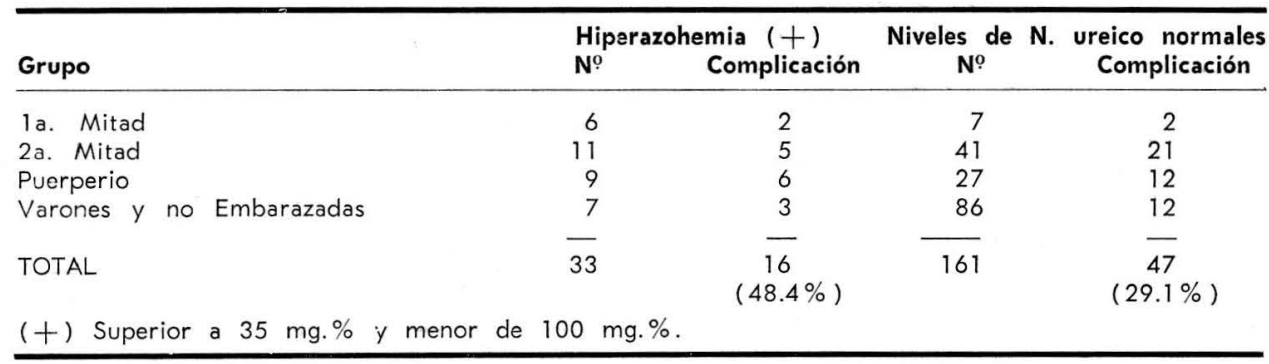

siempre terminan en intervención quirúrgica. En ocasiones el hematoma retroperitoneal conduce a una obstrucción ureteral por fibrosis la cual ha sido denominada por Hutch, Vest y Barlare (cit. 43) "fascis periureteral o periureteritis plástica". Cooper (9), teniendo en cuenta estas contingencias, recomienda no practicar biopsias renales en lugares en donde una intervención quirúrgica no pueda practicarse.

Menos frecuentemente son observadas infecciones del riñón, bacteremias, séptico-piohemias (29, 30, 40, $41,42,45,51)$, extravasación perirenal de orina (45), laceración grave del riñón $(29,30,40,41)$, lesión de otras vísceras como la vesícula y el páncreas (54), neumotórax y anuria (29). En un caso se obtuvo tejido esplénico cuando se realizó una biopsia izquierda, e hígado en doce oportunidades, la mayoría de las veces conjuntamente con tejido renal. Al igual que otros grupos (41, 45, 58), no se observó complicación luego de herir estas vísceras. El cólico renal $(12,27,28,30,33,37,46$, 58) tampoco es frecuente ni reviste mayor gravedad ya que cede con la administración de antiespasmódicos, aunque hay ocasiones que requiere un bloqieo lumbar como única for- ma de calmarlo (44). En algunos pacientes se presenta fiebre por uno o dos días después de la biopsia sin causa que lo explique, pero cede habitualmente en forma espontánea (15, 40, 44). El simple dolor local luego de la punción, considerado por algunos autores como complicación (15, $27,28,37,40,41,44)$, en nuestra opinión no debe ser considerado como tal ya que es una consecuencia lógica que sigue a toda maniobra traumática.

Una contingencia que puede presentarse y que alarma al operador poco experimentado, es la hipotensión brusca y marcada durante el acto de la biopsia. Créese sea debido a sensibilidad a la procaína, a efectos colaterales de la premedicación barbitúrica $\mathrm{y} / \mathrm{o}$ al estado de aprehensión de la paciente. Gadner (16) relata en su experiencia sobre 800 biopsias, haberse hallado ante 15 de estos casos y en todos la presión arterial se normalizó con solo remover la almohada de debajo del vientre. Reprodujo el cuadro colocándola de nuevo. Considera que aunque la etiología de esta hipotensión no está todavía muy clara, la presión ejercitada sobre el vientre en pacientes hipertensos e hiperemotivos fundamentalmente, puede ser la causa desencadenante. 


\section{CUADRO № 7}

\section{Biopsia Renal percutanea - inCidencia de algunas complicaciones}

\begin{tabular}{|c|c|c|c|c|c|c|c|}
\hline Autor & Niopsias $^{\mathrm{H}}$ & $\begin{array}{c}\text { emorragia } \\
\text { + trans- } \\
\text { fusión }\end{array}$ & $\begin{array}{l}\text { grave } \\
\text { Hematoma } \\
\text { perirrenal }\end{array}$ & $\begin{array}{c}\text { Nefrectom } \\
\text { por } \\
\text { hematoma }\end{array}$ & Cólico & $\begin{array}{l}\text { Septi- } \\
\text { cemia }\end{array}$ & Muerte \\
\hline Miatello y col. (73) & 1.000 & 0.1 & 0.7 & - & - & - & - \\
\hline Kark y col. (28) & 500 & 0.2 & 0.6 & - & 2.8 & 0.2 & - \\
\hline Dodge y col. (12) & 205 & 7.6 & 3.8 & 0.9 & - & - & 0.4 \\
\hline Muehrcke y col. (40) & 179 & 0.5 & - & - & - & - & - \\
\hline Kark y col. (27) & 100 & 2.0 & - & - & - & - & - \\
\hline White R. H. R. (58) & 100 & 1.0 & - & - & 1.0 & - & - \\
\hline Parrish y col. (45) & 63 & 1.5 & - & - & - & - & - \\
\hline Kauffman. A. (30) & 45 & 8.8 & - & - & 8.8 & - & 2.2 \\
\hline Rocha y col. & 259 & 3.4 & 0.7 & 0.3 & 1.9 & 0.3 & - \\
\hline
\end{tabular}

Finalmente, deseo anotar que los casos que requieren nefrectomía, sutura renal o ureterolisis son muy poco frecuentes y disminuyen cada vez a medida que se observan más cuidadosamente las contraindicaciones y la experiencia en el procedimiento es mayor. La mortalidad en general, relatada por Dodge (12) luego de una encuesta realizada sobre 4.000 biopsias, no excede del $0.12 \%$ y es mucho menor que la reportada para la biopsia del hígado. En el grupo estudiado no se ha observado ningún caso de muerte y las pacientes que han tenido que intervenirse se han redu-

\section{BIBLIOGRAFIA}

1 ALWALL, N.: "Aspiration biopsy of the kidney". A report of a case of emilcidosis diagnosed through aspiration by opsy of the Ridney. Act. Med. Scand. 143: 430, 1952.

2 ALTCHEK, A.: "Electron microscopy of renal biopsies in toxemia of pregnancy". J. Am. Med. Ass., 175, 791, 1961.

3 BJORNEBOE, M., GORMOEN, C. B. H., IVERSEN, F. and ROASCHOU, F.: "The nefrotic syndrome. The effect of corticotropin (ACTH)". Act. Med. Scand. Supp. 266 : 249,1952

4 BJORNEBOE, M. BRUN, C., GORMSEN, H., IVERSEN, I. andw ROASCHOU, F.: "The nefrotic syndrome". Act. Med. Scand. Supp. 266: 142. 233, 1952. cido a una sutura renal y a una nefrectomía en pacientes no embarazadas.

\section{RESUMEN}

Se refieren las complicaciones inmediatas y tardías observadas en un grupo de pacientes sometidas a la biopsia renal percutánea en diferentes períodos del ciclo grávido puerperal. Se comparan con las halladas en un grupo de pacientes no embarazadas y de varones. Se relatan las indicaciones y contraindicaciones del procedimiento.

5 BRUN, C. GORMSEN, H. HILDEN, T. IVERSEN, I. and ROASCHOU, F.: "Diabetic nephropathy. Kidney biopsy and renal function test". Am. J. Med., 15: 187, July Dec., 1953.

6 BRUN, C. and ROASCHOU, F.: “Kidney biopsies". Am. J. Med., 24: 676, 1958.

7 BURCH, R. P., PEARL, M. A. and STEMBERG, W. H.: "A clinico-pathological study of the nefrotic syndrome". Ann. of Int. Med., 56 (1): 54, Jan., 1962.

8 CASTLEMAN, C. and SMITHWICK, "The relation of vascular disease to the hypertensive state". The New Eng. J. of Med., 239 (20) 729, Nov. 11, 1948.

9 COOPER, W. C.: "Experience in percutaneous renal biopsy at A.U.S. Naval Hospital". J. of the Formosan Med. ass., 61. (3): 213, March 3, 1962. 
10 DENNIS, F. J., SMYTHE, C. M. MCIVER, F. A. and HOWE, H. G.: "Percutaneous renal biopsy in eclampsia". Am. J. of Obst. and Gyn. 87 (3): 364, Oct. 1, 1963.

11 DIECKMANN, W. J., MCCARTNEY, CH. P. and HARROD, J. P. "Kidney biopsies in multiparous patients with vascular renal disease in pregnancy". Am. J. Obst. and Gyn., 75: 634, 1958.

12 DODGE, W. F., DAESCHNER, C. W., BRENAN, J. C., ROSEMBERG, H. S., TRAVIO, L. B., and HOPPS, H. C.: "Percutaneouz reanl biopsy in children". Pediatrics 30 (2) 287 Aug. 1962.

13 EUGENE, A. H.: "Biopsia renal percutánea". Clin. Med. N. Amer., 903, Julio, 1963.

14 FLANAGAN, P. and LIBECK, J. R.: "Renal biopsy observations following recovery from ethylene-glyool nefhrosis". The Am. J. of Clin. Path., 41 (2): 171, Feb., 1964.

15 FOFGREN, S. and SNELLMAN, B.: "Instrument and technique of Kidney biopsy". Act. Med. Scand., 157, Fas. I: 93, 1957.

16 GADNER, K. D. and HILDRETH, E. A. : "Hypotension during percutaneous renal byopsy". J.A.M.A. 186 (6): 599. Nov. 9, 1963.

17 CIBELLI, G. R., FRIED, I., SCOROFITS, A., RAPAPORT, N., PENA, H. Y FOVEIRO, J.: "Correlación de las pruebas funcionales con la biopsia renal percutánea en la gestosis". La Prensa Med. Arg. (24): 1503, Julio 14, 1963.

18 CINSBURG, I. W., DURANT, J. R. and MENDEZ, L.: "Percutaneous renal biopsy under direct radiologic direction". J.A.M.A. 181 (3): 211, July 21, 1962.

19 GREENWALD, H. P., BRONFIN, G. J. and AUERBACH, O.: "Needle biopsy of the kidney. A report of five cases of multiple myeloma". Am. J.. Med. 15: 198, JulyDec. 1953.

20 GUILHEM, P., SUC, J. M., MOREAU, G., PONTONIER, G. et PUTOIS, J.: "La ponction biopse du rein dans les syndromes vasculo-renaux de la grosseasse. Notre expérience personnelle. Notions preliminaires. Bull. Fed. Gynec. Obst. Franc., 14 (5): 798. Nov-Dec. 1962.

21 IVERSEN, P. and BRUN, C.: "Aspiration biopsy of the kidney". Am. J. of Med., 11 : 324, Sep., 1951.
22 JACKSON, J. D., SMITH, F. G., LITMAN, N. N., YINLE, C. L. and LATTS, H.: "The Fanconi Syndrome with cystinosis". The Am. J. Med., 23 (6) : 893 Dec., 1962.

23 JACOBSON, M. H. and NEWMAN, W.: "Study of Pyelonephritis using renal biopsy material". Arch. Of Int. Med. ,110 (2): 211, Aug., 1962.

24 KARK, R. M., POLLACK, V. E., SOOTHILL, J. F., PIRANI, C. L. and MUCHRCKE, R. C.: "Simple test renal function in health and diseases". A N. A. Arch. of Int. Med., 99 (2): 176, Feb., 1957.

25 KARK, R. M., PIRANI, L. POLLACK, V. E. MUEHRCKE, R. C. and BLAINEY, S. O.: "Nephrotic syndrome in adults". Ann. Int. Med., 49: 751, 1958.

26 KARK, R. M., MUEHRCKE, R. C., PIRANI, C. L. and POLLACK, V. E.: "Correlations of serial renal biopsies and other data in patients with the nephrotic syndrome". J. Clin. Invest., 34: 944, 1955.

27 KARK, R. M. and MUEHROKE, R. C.: Biopsy of kidney in prone position". Lancet. 1: 1047, Mayo 22, 1954.

28 KARK, R. M., MUEHRCKE, R. C., POLLAK, V. E., PIRANI, C. L., KIEFER, J. H.: "An analysis of five hundred percutaneous renal biopsies". A.M.A. Arch. Int. Med., 101 : 4391958.

29 KARK, R. H. MUEHRCKE, R. C., PIRANI, C. L. and POLLAK, V. R.: "The clinical value of renal biopsy". Ann. Int. Med. 43 (4) : 807, 1955.

30 KAUFMAN, A. and GUINAN, B. A. "La biopsia renal por punción percutánea. Análisis de 45 casos". Rev. Ven. de Urol., 13 (3) $4: 367$, Julio-Dic. 1961.

31 KINMESTIEL, P., KIM, On. Ja. and BERES, J.: "Studies on renal biopsy specisen with the aid of the electron microscope". The Am. J. of Clin. Path., 38 (3): 270 Sep. 1962.

32 KINMESTIEL, P., KIM. On. Ja. adn BERES J.: "Studies on renal biopsy specimens with the aid of the electron microscope". The Am. J. of Clin. Path., 38 (3): 280, Sep., 1962.

33 KIPNIS, G. P., JACKSON, G. G., DAILEM$\mathrm{BACH}, \mathrm{F}$. D. and SCHOENBERGER, J. A.: Renal biopsy in pyelonephritis. Correlative study of kidney morphology, bacteriology and function in patients with chronic urinary infections". A.M.A. Arch. of Int. Med. 95 (3): 445, March, 1955. 
34 LICH, R.: "Renal biopsy". J.A.M.A. 163 (6): 420, Feb. 9, 1957.

35 LINDANER, N. A. and ITSKOVITZ, H. D. : "Renal Biopsy in hyperaldosteronism". The Am. J. of Card., 14: 553, Oct. 1964.

36 LISTER, L. M. and HAKER, R. D. : "Needle biopsy of the kidney in the diagnosis dsiseminated Lupus Erythematosus". The Am. J. of Med., XVII (96): 851, Dec. 1954.

37 MIATELLO, V. R., MEDEL, R. P., MALEDO, L. I., MORELLI, O. H., COTLIEB, D., PLANS, C. P. y FALCON, C. A.: "Riesgos y valor de la biopsia por punción transcutánea según nuestra experiencia en 1.000 biopsias realizadas". La Prensa Med. Arg. XLVIII (39): 2679, Sep. 29, 1961.

38 MUEHRCKE, R. C., KARK, R. M. PIRANI, L. C. and POLLAK, V. E.: "Lupus nephritis". Medicine, 36: 1, 1957.

39 MUEHRCKE, R. C., KARK, R. M., PIRANI C. L. and SCHOENBERGER, J. A.: "Serial kidney biopsy studies in patients with nephrotic syndrome". J. Lab. and Clin. Med. 44 (6): 901, Dec., 1954.

40 MUEHRCKE, R. C., KARK, R. N. and PIRANI, C. L.: "Biopsy of the kidney in the diagnosis and management of renal disease". The New Eng. J. of Med., 253 (13): 537, Sep. 29. 1955.

41 MUEHRCKE, R. C., KARK, R. M. PIRANI, C. L.: "Technique of percutaneous renal biopsy in the prone position". J. of Urol. 74 (3): 267, Sep. 1955.

42 NETTLES, J. B. and BROWN W. E.: "Renal biopsy in normal and toxemic pregnancy". Clin. Obst. and Gyn. 4 (3): 757, Sep. 1961.

43 OPPENHEIMER, G. D. and GOLDMAN, H.: "Periureteral fibrosis. An unusual complications of renal biopsy". The J. of Urol. 88 (5) : 611, Nov., 1962.

44 PARDO, V., CARDENAS, C. F. y MASO, C. "Biopsia renal por punción". Rev. Clin. Españ. XLIX (6): 279, Junio 30, 1953.

45 PARRISH, A. F. and HOWE, J. S.: "Needle biopsy as aid in diagnosis of renal disease". J. Lab. and Clin. Med., 42: 152, 1953.

46 PIRANI, C. L., MUEHRCKE, R. C. and KARK, R. M.: "Renal diseases as studied by serial kidney biopsy". The Am. J. of Path., XXXI (3): 594, May-Jun. 1955.
47 POLLAK, V. E. and NETTLES, J. B.: "Preliminary observations on the differential diagnosis of toxemias of pregnancy by means of renal biopsy". Am. J. Obst. and Gyn. 79: 866, May, 1960.

48 POLLAK, V. E., PIRANI, C. L. KARK R. M. MUEHRCKE, R. C., FREDA, V. C. and NETTLES, J. B.: "Reversible glomerular lesions in toxemia of pregnancy". Lancet, 2 : 5962,1956

49 ROLAND, A. S. and DIMOND, E. G. : "The value of percutaneous renal biopsy in the hypertensive subjet". Am. Heart J. 66 (1) 140 July, 1963.

50 RUPE, C. R. EYLER, W. R. and IRWIN, J. L. : "Localization of kidney for renal biopsy". The J. of Urol. 88 (4): 455, Oct. 1962.

51 SAMELLAS, W.: "Death due to septicemia following percutaneous needle biopsy of the kidney". The J. of Urol., 91 (4): 317, April, 1964

52 SCHOWITZ, L. J. POLAK, V. E., PIRANI, C. L. and STEIN, P.: "Pre-eclampsia and eclampsia early in pregnancy. Study of nine cases by renal biopsy". Am. J. of Obst. and Gyn., 84 (10): 1330, Nov. 15, 1962.

53 SiEgleR, A. M., MALFETANO, J.: "The pathofgenesis of puerperal anuria and the value of renal biopsy examination". Am. J. of Obst. and Gyn. 69 (6): 1217, Jan. 1955.

54 ELOTKIN, E. A. and MADSEN, P. O.: "Complications of renal biopsy. Incidence in 5.000 reported cases". The $\mathrm{J}$. of Urol. 87 (1): 13, Jan. 1962.

55 SMETANA, H. F.: "The needle biopsy in diagnosis". Am. J. Clin. Path. 24 (4): $395,1954$.

56 VERTES, V. and GRAUL, J. A.: "Observations on renal hypertension. The role of renal biopsy". Circulating, XXVIII (4): 536, Oct. 1963.

57 WEDGWOOD, R. J. P. and KLAUS, M. H.: "Anaphylactoid purpura (Schonlein Henoch Syndrome): long tara following up study wiht special reference to renal involmement". Pediatrics, 16: 196, 1955.

58 WHITE, R. H. R.: "Observations on percutaneous renal biopsy in children". Arch. Dis. Chil, 38 (199): 260, June, 1963.

59 ZELMAN, S.: "Fatal hemorrhage following needle biopsy in uremia". J.A.M.A. 154 (12): 997, March. 1954. 